\title{
LUT
}

University

\section{NMR Relaxation of Functionalized Dendrimers}

Sheveleva Nadezhda N., Dolgushev Maxim, Lähderanta Erkki, Markelov Denis A.

This is a Post-print version of a publication

published by ACS Publications

in Macromolecules

DOI: 10.1021/acs.macromol.9b01427

Copyright of the original publication: () 2019 American Chemical Society

Please cite the publication as follows:

Sheveleva, N. N., Dolgushev, M., Lähderanta, E,, Markelov, D. A. (2019). NMR Relaxation of Functionalized Dendrimers. Macromolecules, vol. 52, issue 24. pp. 9766-9772. DOI: 10.1021/ acs.macromol.9b01427

This is a parallel published version of an original publication.

This version can differ from the original published article. 


\title{
NMR Relaxation of Functionalized Dendrimers
}

Nadezhda N. Sheveleva, ${ }^{1,3}$ Maxim Dolgushev, ${ }^{2 *}$ Erkki Lähderanta, ${ }^{3}$

Denis A. Markelov ${ }^{1}$

${ }^{1}$ St. Petersburg State University, 7/9 Universitetskaya nab., St. Petersburg, 199034 Russia.

${ }^{2}$ Sorbonne Université, CNRS, Laboratoire de Physique Théorique de la Matière Condensée, LPTMC, 75005 Paris, France.

${ }^{3}$ Department of Physics, LUT University, Box 20, 53851 Lappeenranta, Finland.

*dolgushev@lptmc.jussieu.fr

\begin{abstract}
Functionalized dendrimers are a novel type of dendritic macromolecules that contain functionalized groups that do not take part in the dendrimer's synthetic cycle. Focusing on the local dynamics of the functionalized dendrimers we study the NMR relaxation by considering the spin-lattice relaxation rate $1 / T_{1}(\omega)$ of the ${ }^{1} \mathrm{H}$ and ${ }^{13} \mathrm{C}$ nuclei and the corresponding spectral density. The macromolecules are modeled in the framework that accounts for the local bending rigidity, which is of paramount importance for the NMR relaxation of dendritic structures. We show that the behavior of the NMR relaxation of the functionalized dendrimers is qualitatively different from that of the standard dendrimers manifested through an additional maximum in $\omega / T_{1}(\omega)$. The new theoretical picture has allowed us to analyze recent atomistic molecular dynamics simulations of carbosilane dendrimer
\end{abstract}


melts and to elucidate the differences in the high-frequency behavior of their spinlattice relaxation rates from the predictions of the standard dendrimer model. 


\section{Introduction}

Dendrimers represent a particular kind of macromolecules that have a series of advantageous features such as the presence of a large number of terminal groups, perfect symmetry, compactness, etc. ${ }^{1}$ These features are used in a variety of applications, for example, in delivery of $\operatorname{drugs}^{2}$ or dyes ${ }^{3}$ and in the synthesis of metal or magnetic nanoparticles. ${ }^{4}$ Possible benefits from the dendrimers' properties for the improvement of the efficiency of solar cells, ${ }^{5}$ and of the viscosity properties of complex liquids, ${ }^{6}$ are actively investigated.

The inner space of a dendrimer is actively used in many applications. This is related to their possibility to provide an inner space for encapsulated matter. ${ }^{1}$ One of the most important parameters of this feature is the adsorption/desorption time of the hosted matter, which depends on the local mobility of macromolecules. ${ }^{7}$ Therefore the development of the theory of dendrimers' local mobility is a fundamental question that is important for the enhancement of the technologies involving dendrimer macromolecules.

NMR relaxation is perhaps one of the most effective methods for studying local dynamics in macromolecules. ${ }^{8,9}$ It provides access to the local reorientation mobility without perturbing the investigated system. ${ }^{10}$ It turned out that for dendrimers, as opposed to linear chains, traditional approaches, in which shorter spin-lattice relaxation times correspond to less mobile nuclei, are not appropriate for the analysis of the mobility of NMR active groups (see Ref. ${ }^{11}$ for details). Therefore this question has been investigated by means of theoretical-analytical, ${ }^{12-}$ 
14 numerical, ${ }^{15-17}$ and experimental ${ }^{11,18-22}$ methods. Nowadays, the picture of dendrimer relaxation appears to be clarified in detail. ${ }^{23}$ It has been shown that the following three processes have the main contribution to the relaxation in dendrimers: (1) the local mobility of dendrimers' segments represented through spatially periodic modes, (2) the relaxation of dendrimers' branches as a whole, and (3) the overall dendrimer rotation. Process (1) comprises structurally periodic modes related to the inner oscillations that are characterized by short relaxation times building the so-called inner spectrum. ${ }^{24}$ On the contrary, the modes representing process (2) involve the synchronized movement of all the beads contained in the branch. ${ }^{13,25,26}$ Later in the text, we use the term "branch relaxation process" for designating process (2). Process (3) has not yet been captured by analytical theory, albeit the first steps towards overhauling this issue have been performed in recent work. ${ }^{27}$ Nevertheless, in the case of a dendrimer melt, this process is shifted towards very low frequencies, which has allowed the bridging of analytical theory and computer simulations. ${ }^{28}$

In particular, it was shown that the bending rigidity plays a fundamental role in the NMR relaxation of dendrimers. Neglecting this aspect leads to the spurious result that the NMR relaxation of flexible dendrimers only depends on the local mobility of segments ${ }^{15}$ (i.e., only on process (1), which is independent of the location of the labeled segment in the macromolecule and of the size of macromolecule). On the contrary, for semiflexible dendrimers, the NMR relaxation rates of the labeled segments strongly depend on their remoteness from the 
dendrimer's periphery ${ }^{13}$ (i.e., on process (2) related to the relaxation of the dendrimer's branch originating from the labeled segment). It is important to note that for other dynamical characteristics, such as mechanical relaxation, other aspects, such as restricted size fluctuations, could be important. ${ }^{29}$ The abovementioned results apply to the dendrimers with an ideal dendritic structure. However, the progress in the dendrimer synthesis has yielded novel dendritic structures (see e.g., Ref. ${ }^{30}$ for a review), among them pseudodendrimers ${ }^{31,32}$ and functionalized dendrimers. ${ }^{33-35}$ Unlike dendrimers, pseudodendrimers are not perfectly structured macromolecules and have irregularly distributed branching points and different length of the chains between two branching points. ${ }^{31,32}$
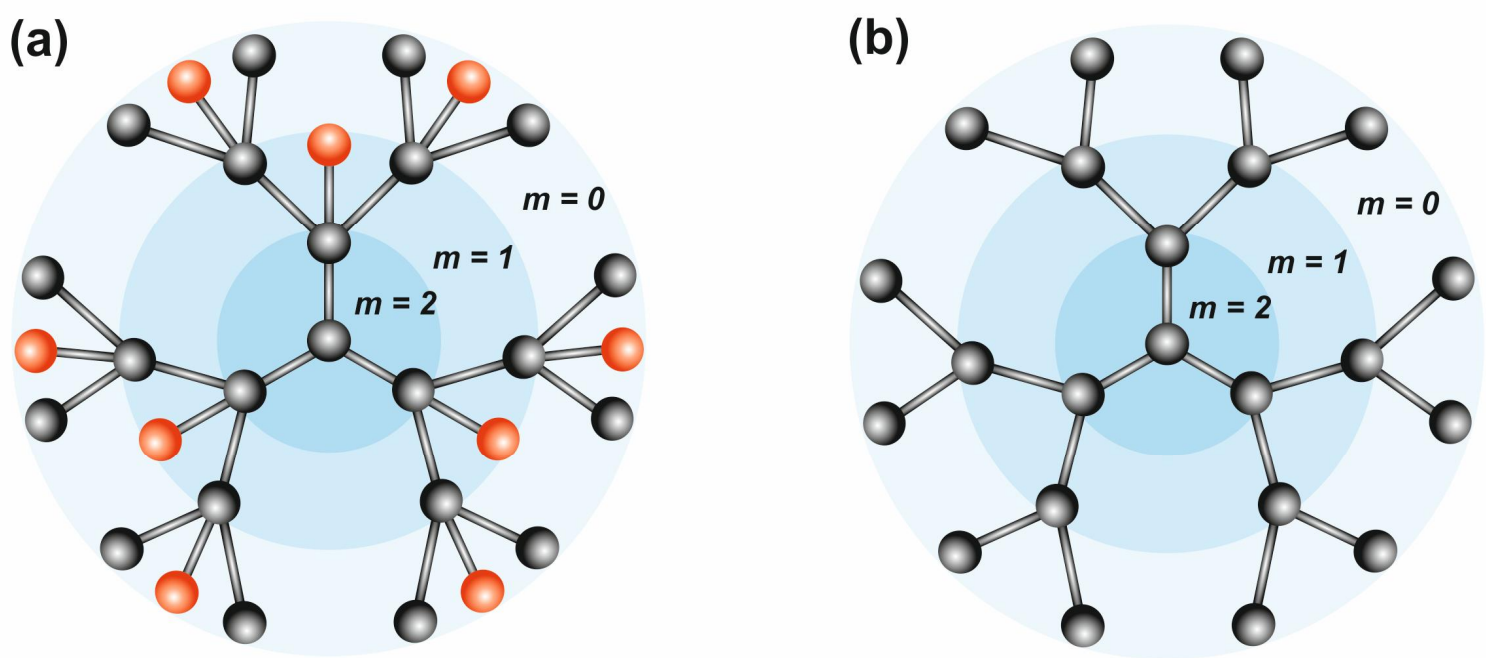

Figure 1. Schematic drawing of an internally functionalized (a) and a standard (b) dendrimers, both of generation $G=3$. The functional beads in (a) are depicted in red. Also, we indicate by $m$ the shells of the dendrimers from periphery to core.

In this work, we theoretically investigate the NMR relaxation of the functionalized dendrimers (Figure 1), in which additional segments at the 
branching points appear. The first step towards a theoretical description of the dynamics of the functionalized dendrimers was done in work by Grimm and Dolgushev (GD) ${ }^{36}$ There, it was established that the presence of the functionalized segments leads to a new process (we will call it "GD-process" in the following), which does not exist for standard dendrimers. The GD-process comprises new asymmetric normal modes involving the motion of functionalized segments and branches that grow from the same branching point as the functionalized segments. ${ }^{36}$ These additional modes can significantly affect the characteristics of dynamics. For instance, remarkable effects are observed in the dielectric relaxation of the labeled segments originating the branches that are neighbors of the functionalized segments. In this context, a question about the role of the GDprocess for the NMR relaxation of the functionalized dendrimers arises. To answer it we consider the dynamics of the functionalized dendrimers focusing both on the models with and without bending rigidity; taking it into account turns out to be important for capturing the specificity of functionalized dendrimers. 


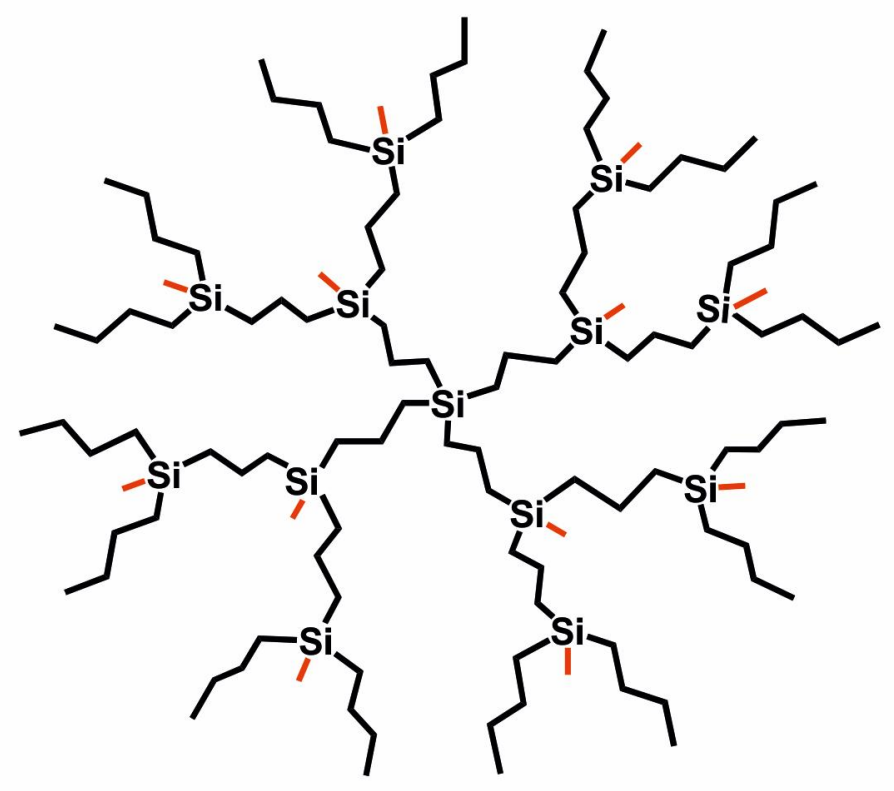

Figure 2. Structure of a carbosilane dendrimer of the third generation. Note that a $\mathrm{CH}_{3}$ group is attached (red) to each Si-atom except the core.

Furthermore, the present study provides also an explanation of the effects observed in the NMR relaxation in carbosilane dendrimers, studied recently in Ref. $^{28}$, which could not be covered within the theory of standard dendrimers. ${ }^{13}$ While the simulations of Ref. ${ }^{28}$ show good agreement with standard theory in the low frequency region, at high frequencies it was observed that (i) the minimal time characterizing the mobility of terminal segments is around two times smaller than that of the inner segments; (ii) the amplitude of the maximum of the reduced spinlattice relaxation rate in the susceptibility representation $\left[\omega / T_{1 H}\right]$ at high frequencies is considerably higher than predicted by the standard model. As we show in this work, the particularity of the structure of carbosilane dendrimers (that to each $\mathrm{Si}$ atom at the branching points (except the core) a $\mathrm{CH}_{3}$-group is attached, see Figure 2) makes their behavior at high frequencies close to that of 
functionalized dendrimers. Thus, with this, we demonstrate that the NMR relaxation allows the capturing of dynamical features caused by fine particularities in the structure of macromolecules (related to their connectivity; for chemical details atomistic molecular dynamics simulations remain indispensable). Therefore the obtained theoretical results can inspire one to extend the NMR techniques used for standard dendrimers ${ }^{19}$ on functionalized dendrimers. Also these results can be considered as first steps towards the macromolecules with irregular branching. ${ }^{37}$

The paper is structured as follows: In Sec. II we recall briefly the theoretical framework of Ref. ${ }^{38}$ within which we study in Sec. III the NMR relaxation of functionalized dendrimers. In Sec. III we also analyze the results of molecular dynamics simulations of carbosilane dendrimer melts. The paper is closed by the conclusions.

\section{Theoretical Methods}

The dynamics of dendrimers is modeled within the framework of semiflexible treelike polymers, ${ }^{38}$ where the polymer is represented by Gaussian segments (denoted by $\left\{\mathbf{d}_{a}\right\}$ ). The segments have a zero mean $\left\langle\mathbf{d}_{a}\right\rangle=\mathbf{0}$ and the mean-square average length $\left\langle\left|\mathbf{d}_{a}\right|^{2}\right\rangle=l^{2}$. The semiflexibility is introduced through the segment-segment correlations: For two directly connected segments, one has $\left\langle\mathbf{d}_{a} \cdot \mathbf{d}_{b^{\prime}}\right\rangle= \pm l^{2} q$, where $q$ is the stiffness parameter and the plus sign is taken for a head-to-tail orientation of $\mathbf{d}_{a}$ and $\mathbf{d}_{b}$ and is minus otherwise. If the stiffness parameter $q=0$, the polymer is fully flexible. Here, we consider standard flexible dendrimers (FD) and functionalized flexible dendrimers (FFD). For $q \neq 0$ the 
structures are semiflexible. Here we consider that for standard semiflexible dendrimers (SD) and functionalized semiflexible dendrimers (FSD) the values of $q$ follow the choice of Ref. ${ }^{36}$, by taking $q=0.32$ for beads having four nearest neighbors and $q=0.48$ for beads having three nearest neighbors. With these ingredients one can construct the potential energy of the macromolecules and write down the corresponding set of Langevin equations for the motion of dendrimer's beads located in $\left\{\mathbf{r}_{i}\right\}$ in three-dimensional space (see Ref. ${ }^{38}$ for details):

$$
\zeta \frac{\partial}{\partial t} \mathbf{r}_{i}(t)+\frac{3 k_{B} T}{l^{2}} \sum_{j} A_{i j} \mathbf{r}_{j}(t)=\mathbf{w}_{i}(t)
$$

where $\zeta$ is the friction coefficient, $k_{B}$ is the Boltzmann constant, $T$ is the temperature, and $\mathbf{w}_{i}(t)$ is the white noise, $\left\langle\mathbf{w}_{i}(t) \cdot \mathbf{w}_{j}\left(t^{\prime}\right)\right\rangle=6 k_{B} T \zeta \delta_{i j} \delta\left(t-t^{\prime}\right)$. Note that the combination of the above-mentioned parameters, $\tau_{0}=\zeta l^{2} /\left(3 k_{B} T\right)$, represents the characteristic relaxation time of an isolated segment (dimer). ${ }^{39}$ The matrix $\mathbf{A}=\left(A_{i j}\right)$ encodes the architecture and semiflexibility of the dendrimers, whose general structure can be found elsewhere. ${ }^{38}$ Solving the set of Langevin equations (1) by the diagonalization of matrix $\mathbf{A}$ (i.e., by the normal mode transformation $\mathbf{r}_{i}(t)=\sum_{k} Q_{i k} \mathbf{u}_{k}(t)$; see Ref..$^{40}$ and Ref. ${ }^{36}$ for the modes of SD and of FSD, respectively), it is straightforward to consider the dynamics of the segments (say, $\mathbf{d}_{a}(t)=\mathbf{r}_{i}(t)-\mathbf{r}_{j}(t)$ that directly connects two beads located at $\mathbf{r}_{i}(t)$ and $\left.\mathbf{r}_{j}(t)\right)$. The corresponding segmental time-autocorrelation function $M_{1}^{a}(t)$ $=\left\langle\mathbf{d}_{a}(t) \cdot \mathbf{d}_{a}(0)>/ l^{2}\right.$ is given by

$$
M_{1}^{a}(t)=\sum_{k} C_{k}^{a} \exp \left[-t / \tau_{k}\right]
$$


Here $\left\{\tau_{k}\right\}$ are the relaxation times related to the full set of distinct nonzero eigenvalues $\left\{\lambda_{k}\right\}$ of the matrix $\mathbf{A}$ by $\tau_{k}=\tau_{0} / \lambda_{k}$. Furthermore, $C_{k}^{a}$ are the coefficients reflecting the contribution of the normal modes $\left\{\mathbf{u}_{k}(t)\right\}$ to the $M_{1}^{a}(t)$ function. Thus, the corresponding relaxation times $\left\{\tau_{k}\right\}$ play a different role depending on the location of the labeled segment $\mathbf{d}_{a}(t)$ in the dendrimer, as we illustrate in Figure 3. As can be inferred from the figure (and was shown in the analysis of the normal modes ${ }^{24-26,36,40}$ ), for standard dendrimers the relaxation processes are associated either with the inner relaxation (related to normal modes of the spatially periodic kind ${ }^{24,26,40}$ ) or the branch relaxation (related to normal modes of the spatially exponential kind ${ }^{24,26,40}$ ); for functionalized dendrimers the GD process (related to normal modes, in which the motion of a functionalized segment excites the neighboring branch ${ }^{36}$ ) also comes into play. Accounting for local stiffness (which is indispensible for a correct description of the NMR relaxation of dendrimers ${ }^{13}$ ) leads to a significant suppression of the inner relaxation, whereas the GD process conserves its significance. 


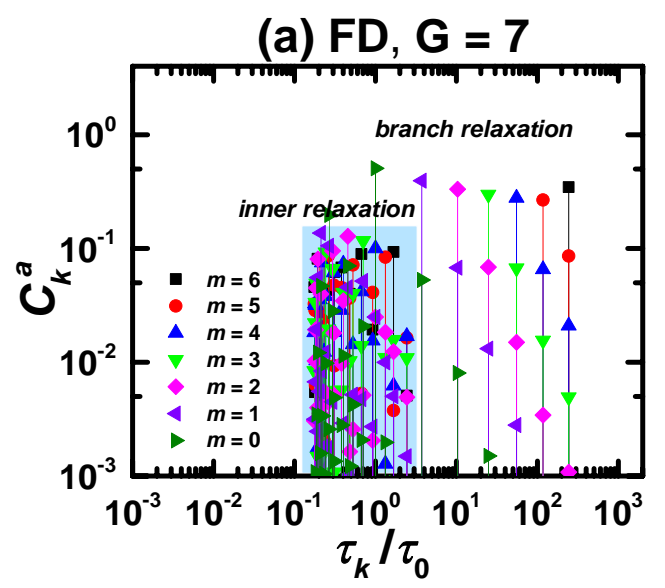

(c) FFD, G = 7

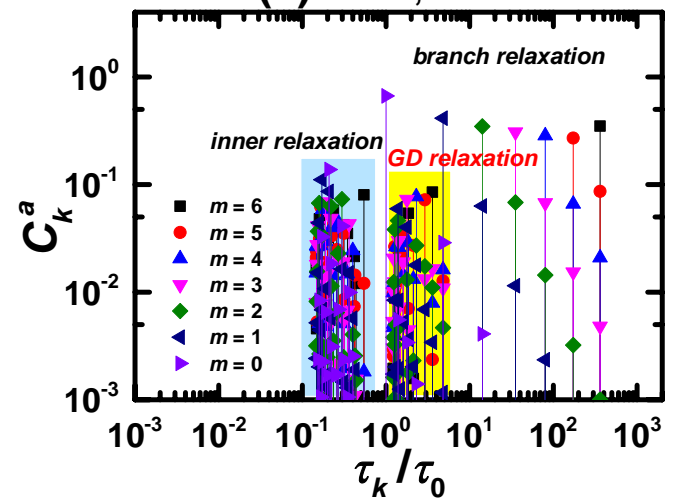

(b) SD, G = 7

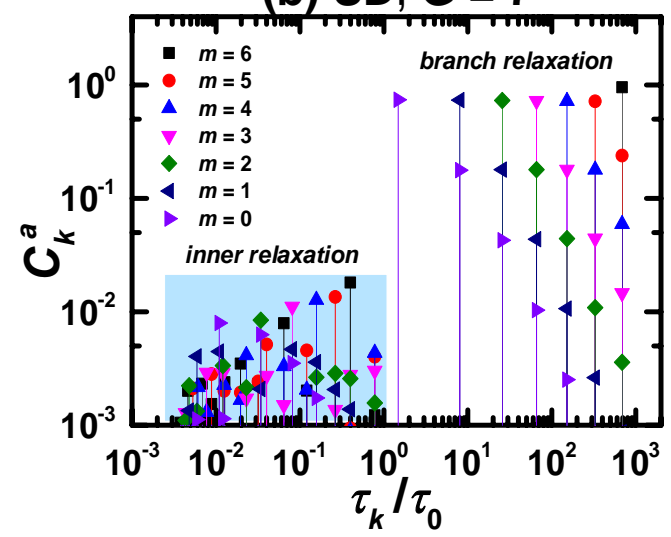

(d) FSD, G = 7

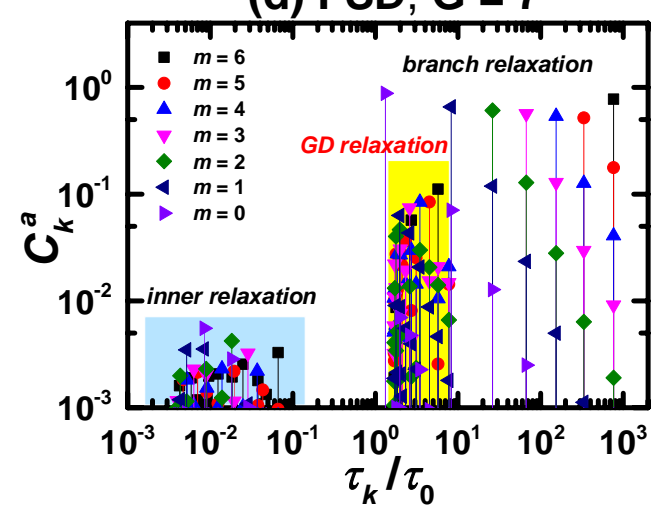

Figure 3. Normal modes' decay contributions $C_{k}^{a}$ in $M_{1}^{a}(t)$, Eq. (2), vs relaxation times $\left\{\tau_{k}\right\}$ for standard (a) flexible (FD), (b) semiflexible (SD) dendrimers and functionalized (c) flexible (FFD), (d) semiflexible (FSD) dendrimers of generation $G=7$. For standard dendrimers the spectrum is split between the inner relaxation at short times and the branch relaxation at long times. For functionalized dendrimers an additional process of the GD relaxation appears.

The function $M_{1}^{a}(t)$ is fundamental for the determination of the spectral density,

$$
J(\omega)=\int_{0}^{\infty} P_{2}^{a}(t) \cos (\omega t) \mathrm{d} t
$$

of the second Legendre polynomial $P_{2}^{a}(t)$ that, for Gaussian $\left\{\mathbf{d}_{a}\right\}$, is given by ${ }^{41}$

$$
P_{2}^{a}(t)=1-3\left\{x^{2}-\frac{\pi}{2} x^{3}\left[1-\frac{2}{\pi} \arctan x\right]\right\} \text {, with } x=\frac{\sqrt{1-\left[M_{1}^{a}(t)\right]^{2}}}{M_{1}^{a}(t)}
$$


The spectral density $J(\omega)$ gives access to the reduced NMR spin-lattice relaxation rates of the ${ }^{1} \mathrm{H}$ and ${ }^{13} \mathrm{C}$ nuclei in the susceptibility representation, ${ }^{10,42}$

$$
\left[\frac{\omega}{T_{1 \mathrm{H}}}\right]=\omega_{\mathrm{H}}\left(J\left(\omega_{\mathrm{H}}\right)+4 J\left(2 \omega_{\mathrm{H}}\right)\right)
$$

and

$$
\left[\frac{\omega}{T_{1 \mathrm{C}}}\right]=\omega_{\mathrm{C}}\left(6 J\left(\omega_{\mathrm{C}}+\omega_{\mathrm{H}}\right)+J\left(\omega_{\mathrm{C}}-\omega_{\mathrm{H}}\right)+3 J\left(\omega_{\mathrm{C}}\right)\right)
$$

respectively, where the angular resonance frequencies $\omega_{\mathrm{H}}$ and $\omega_{\mathrm{C}}$ are related to each other through the gyromagnetic ratios $\gamma_{\mathrm{H}}$ and $\gamma_{\mathrm{C}}$ by $\omega_{\mathrm{C}} / \omega_{\mathrm{H}}=\gamma_{\mathrm{C}} / \gamma_{\mathrm{H}} \approx 0.25$.

Note, that in experiments such as field-cycling ${ }^{1} \mathrm{H} \mathrm{NMR},{ }^{21,22}$ the $\left[\omega / T_{1 H}\right]$ function includes both intra- and intermolecular mobility contributions. For instance, as established in Ref. ${ }^{21}$ by experimental studies of poly(propyleneimine) dendrimer melts, this function is determined by local dynamics at high frequencies, breathing modes (i.e., branch relaxation) at intermediate frequencies, and overall tumbling (i.e., rotation of the dendrimer as a whole), and diffusion at low frequencies. The intermolecular processes have a significant contribution at low frequencies. ${ }^{21}$ Molecular dynamics simulations of poly(butilcarbosilane) dendrimer melts reached similar conclusions. ${ }^{28}$ However, it was established that the contribution of rotation of the dendrimer as a whole to a segmental reorientation is smaller than $3 \% .^{28}$ Therefore, notwithstanding the fact that the theoretical model does not take into account the intermolecular contribution, it is suitable to capture the specificity of the functionalized dendrimers. Indeed, as we proceed to show in 
Section III, their hallmark - the GD process - appears in the high-frequency region, where the intermolecular contribution to $\left[\omega / T_{1 H}\right]$ is minimal.

\section{Results and Discussion}

\section{A. Spectral Density}

The spectral density $J(\omega)$, in Eq. (3), carries information about the NMR relaxation functions, as given by Eqs. (5)-(6). ${ }^{10}$ Therefore we start with a discussion of $J(\omega)$ converted into the susceptibility representation, ${ }^{42,43}$

$$
[J(\omega)]=\omega J(\omega),
$$

see Figure 4 for functionalized flexible (FFD) and semiflexible (FSD) dendrimers. The results are obtained through Eq. (3) by numerical integration (using the discrete-time Fourier transform method with fixed time step $10^{-5} \tau_{0}$ ) for different segments of generation $G=3, \ldots, 7$ dendrimers. The segments are numbered from the periphery by index $m$ so that the terminal segments always have $m=0$. Note that with $m>0$ we denote the internal segments only (i.e., not the functionalized ones). In this case, as can be observed in Figure 4, the qualitative differences between FFD and FSD are very strong. Also, we stress that for all dendrimers the spectral densities are superimposed at constant $m$ but different $G$, except for the core segments $(m=G-1)$, to which no functionalized groups are connected. Therefore, the feature that the NMR rates of standard dendrimers are determined by the remoteness of the labeled segment from the periphery ${ }^{13}$ remains valid for functionalized dendrimers. The exceptional case of $m=G-1$ is related to the fact 
that these segments seed the largest possible branch so that they do not have a neighboring segment belonging to a larger branch, whose relaxation is important to the segment of the $m$ th shell. This feature was also observed for standard dendrimers and is more important for semiflexible macromolecules. ${ }^{13}$

(a) FFD

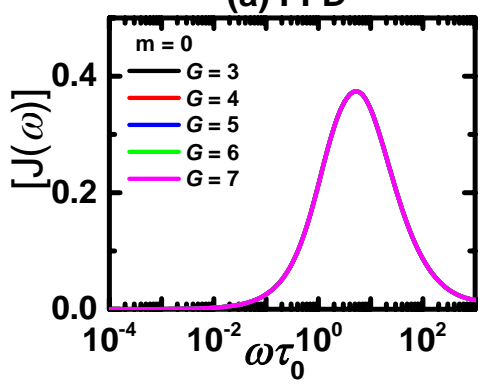

(d) FSD (b) FFD

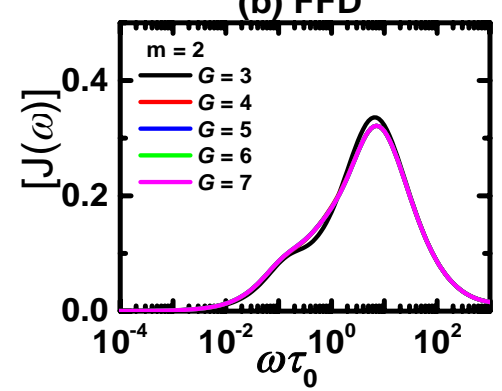

(e) FSD (c) FFD

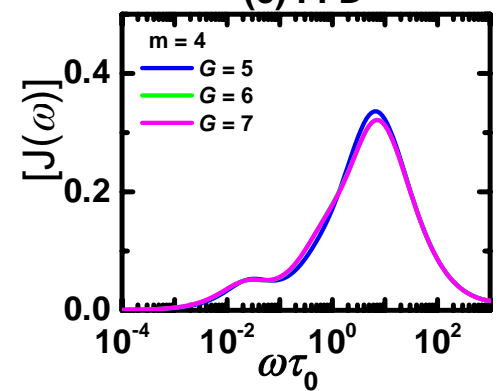

(f) FSD
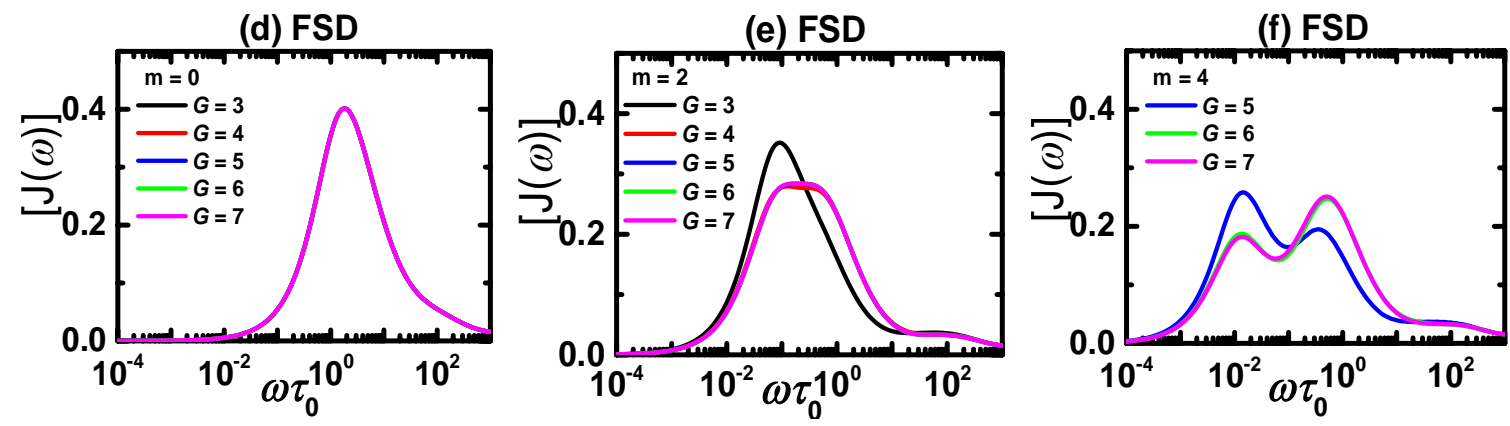

Figure 4. Function $[J(\omega)]$, Eq. (7), for segments of flexible $(a, b, c)$ and semiflexble ( $d, e, f)$ functionalized dendrimers (FFD and FSD, respectively). The dendrimers' generation is labeled by G. Note that the curves are superimposed for the segments having the same index $m$. Only the curves with $m=G-1$ deviate from the others.

Given the universality of the behavior of $[J(\omega)]$ at the same value of $m$, we focus in the following on the dendrimers at the highest generation $G=7$, see Figure 5 for FFD and FSD. As can be inferred from the figure, there are strong differences in the behavior of the two systems. For FFD one can see two maxima for almost all 
curves. The main maximum remains at the same frequency for different $m$, as in the case of the standard dendrimer. ${ }^{13}$ The second maximum observed at $10^{-1}-10^{-3}$ $\omega \tau_{0}$ is shifted towards lower frequencies for higher values of $m$. At $m=1$ and 2 this process leads to a broadening of the main maximum and for $m \geq 3$ the second maximum is clearly resolved. The dominance of the high-frequency process resembles the behavior observed for standard flexible dendrimers (FD) in Refs. ${ }^{13-}$ 15, and we note that the GD process is not explicitly observed for FFD.
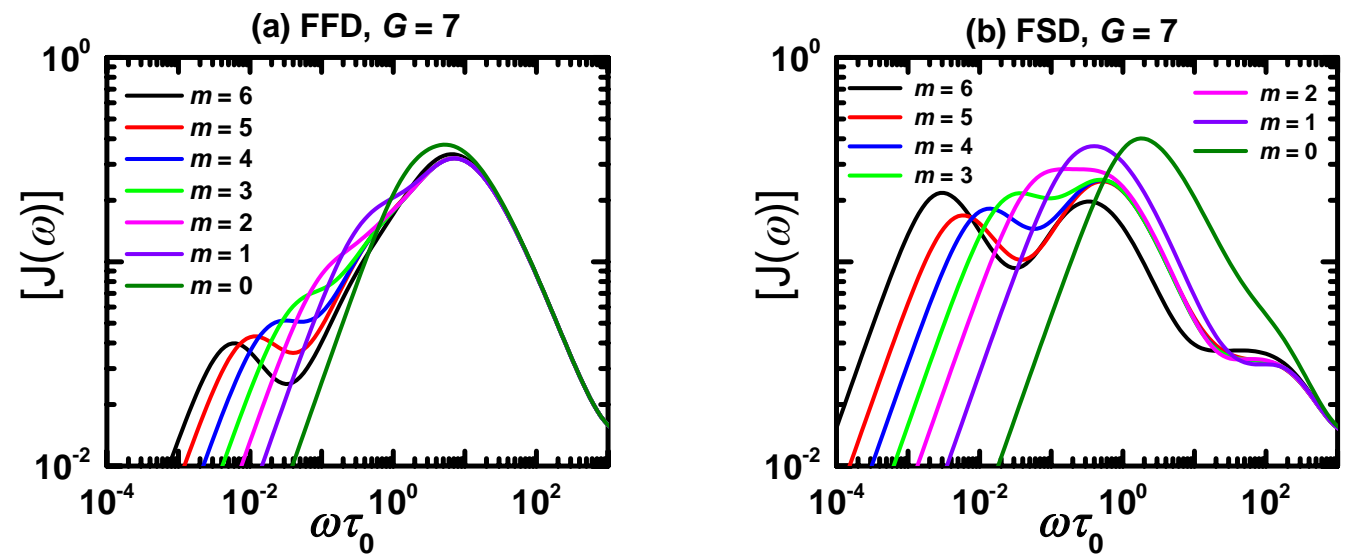

Figure 5. Function $[J(\omega)]$, Eq. (7), for segments labeled by $m$ for functionalized flexible $(F F D)(a)$ and semiflexible $(F S D)(b)$ dendrimers at generation $G=7$.

Turning now to the spectral density of FSD that are locally stiff, Figure 5(b), we observe that for $m \geq 3$ two maxima with comparable heights clearly manifest. As for FFD, the low-frequency maximum is related to relaxation of the dendrimer's branches which are moving as a whole. Its position is shifted towards low frequencies with growing $m$, given that the corresponding maximal relaxation time of the branch originating from the labeled segment grows exponentially with $m$. The position of the second maximum is practically independent of $m$ (except for 
$m=0$ ), and is observed approximately at $\left(\omega \tau_{0}\right) / 2$. For $m=3$ to 5 both its position and amplitude are conserved. Except in case of $m=0$, the second maximum appears due to the GD process, which is the hallmark of the relaxation of functionalized dendrimers. ${ }^{36}$ We note that this process does not exist for standard dendrimers, for which the second maximum is absent. ${ }^{13}$ For $m=2,[J(\omega)]$ has one flat maximum, showing that the characteristic relaxation time of the branch as a whole originating from this segment, which approaches the GD process. For $m=1$ both processes are practically superimposed leading to a single maximum. Finally, the GD process is absent from $[J(\omega)]$ for $m=0$, showing that the maximal relaxation time of a terminal segment is smaller than that of the GD process; the modes excited by the functionalized groups do not contribute to the relaxation of the terminal segments. ${ }^{36}$

At even higher frequencies (Figure $5(\mathrm{~b})$ ), of order $\omega \tau_{0} \sim 10^{2}$, one can observe the contribution of the inner spectrum (see Figure 3) through a shoulder in $[J(\omega)]$, which weakly depends on the parameters $m$ and $G$. An analogous effect has been observed for standard dendrimers. ${ }^{13,14,23}$ The small amplitude of the almost absent maximum reflects the small contribution of the inner process (i.e., of the decays in Eq. (2) related to the inner relaxation times, see Figure 3), as was the case for SD, in contrast to $\mathrm{FD} .^{13}$

For the sake of direct comparison between functionalized and standard dendrimers, we present $[J(\omega)]$ for both dendrimers in Figure 6. Part (a) concerns the flexible macromolecules (FD and FFD) and part (b) the semiflexible ones (SD 
and FSD). In both cases, one can see that the position of the low-frequency maximum, related to the overall branch relaxation, is practically the same for functionalized and standard dendrimers. However, for flexible dendrimers (FD and FFD), in Figure 6(a), the amplitudes are quite low; for them the peak at higher frequencies dominates. It is related to the inner spectrum, which for FFD is very close to the GD process (see Figure 3(c)). On the contrary, for FSD and SD, in Figure 6(b), the contribution of the inner spectrum is small and shifted to high frequencies, and appears as a small high-frequency shoulder at $\omega \tau_{0} \sim 10^{2}$. At the same time, the relaxation times associated with the GD-process always remain between the two smallest branch relaxation times. ${ }^{36}$ This fact "releases" the GD process (especially for higher $m$ ), which then can be clearly observed for FSD: in the region of frequencies between $0.2 \omega \tau_{0}$ and $2 \omega \tau_{0}$ one can clearly see in Figure 6(b) the GD process for FSD (for $m>0$ ), and its absence for SD.

(a)

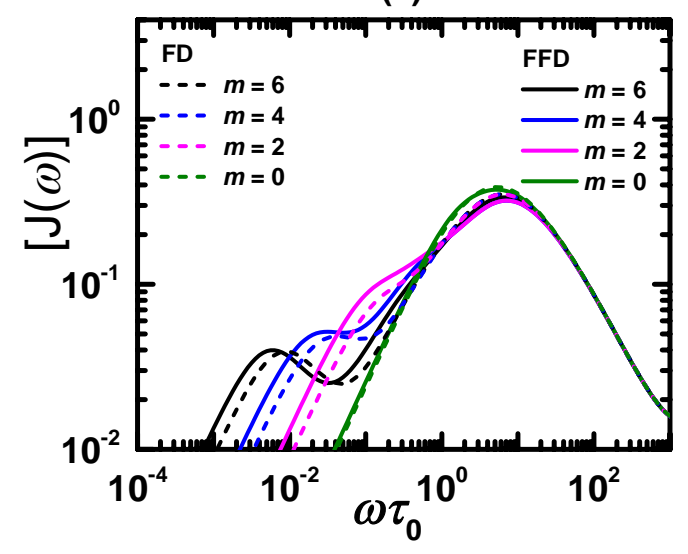

(b)

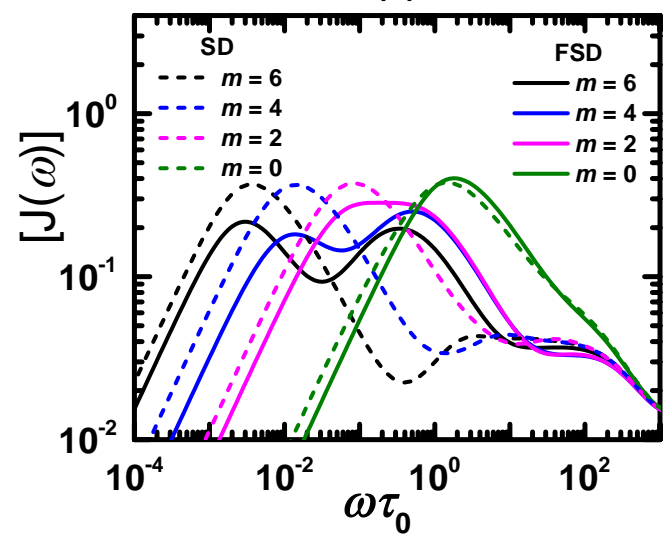

Figure 6. Function $[J(\omega)]$, Eq. (7), for segments of standard flexible (FD) and functionalized flexible (FFD) (a) dendrimers and segments of standard 
semiflexible (SD) and functionalized semiflexible (FSD) (b) dendrimers at generation $G=7$ for different values of $m$.

\section{B. Spin-lattice NMR relaxations}

Here we consider the frequency-dependent spin-lattice relaxation rates $\left[\omega / T_{1 \mathrm{H}}\right]$ and $\left[\omega / T_{1 \mathrm{C}}\right]$ for ${ }^{1} \mathrm{H}$ and ${ }^{13} \mathrm{C}$ nuclei. These functions are determined based on the spectral density $[J(\omega)]$, Eqs. (5)-(6), and they are of experimental interest. ${ }^{10}$ In Figure 7 we present $\left[\omega / T_{1 \mathrm{H}}\right]$ and $\left[\omega / T_{1 \mathrm{C}}\right]$ for FFD and FSD at generation $G=7$. As can be expected; these functions have a qualitatively similar behavior with $[J(\omega)]$ of Figure 5. The positions of the main maxima of $[J(\omega)],\left[\omega / T_{1 \mathrm{H}}\right]$, and $\left[\omega / T_{1 \mathrm{C}}\right]$ for FFD are caracterized by the ratios $\omega_{\jmath}^{\max } / \omega_{H}^{\max } \approx 0.63$ and $\omega_{\jmath}^{\max } / \omega_{C}^{\max } \approx 0.71$, which are close to those defined by the one-process relaxation: $\omega_{J^{\max }}^{\operatorname{ma}} / \omega_{H}^{\max } \approx 0.62$ and $\omega_{J^{\max }} / \omega_{C}{ }^{\max } \approx 0.79$ (see, for instance, Ref. ${ }^{44}$ for ${ }^{1} \mathrm{H}$ and $\mathrm{Ref} .{ }^{45}$ for ${ }^{13} \mathrm{C}$ ). These weak differences can be traced back to the multi-process character of relaxation; here the function $\left[\omega / T_{1 \mathrm{C}}\right]$ turns out to be more sensitive to the presence of additional processes. Finally, we note that the $\left[\omega / T_{1 \mathrm{H}}\right]$ and $\left[\omega / T_{1 \mathrm{C}}\right]$ functions for FSD carry the behavior of the corresponding spectral density $[J(\omega)]$, as discussed in the previous subsection. 
(a) FFD, $G=7$

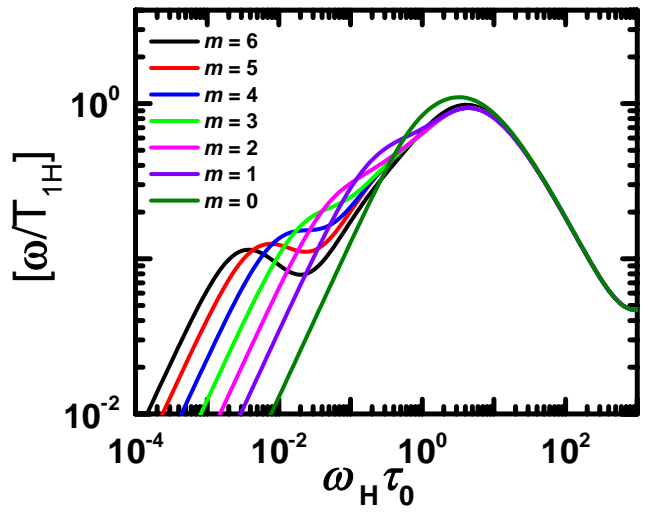

(c) FSD, $G=7$

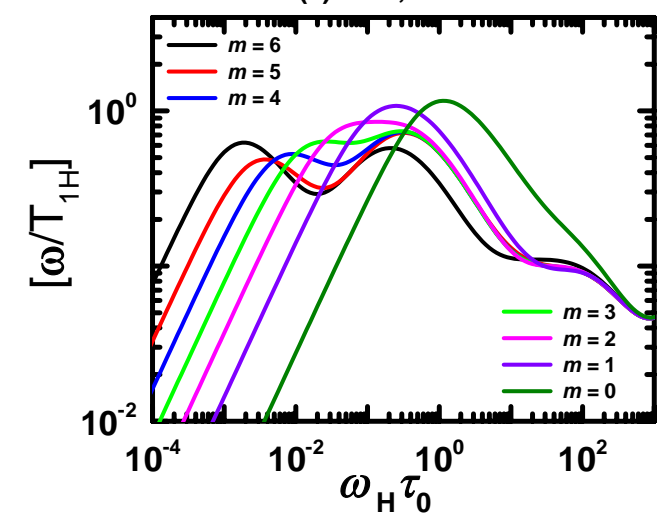

(b) FFD, $G=7$

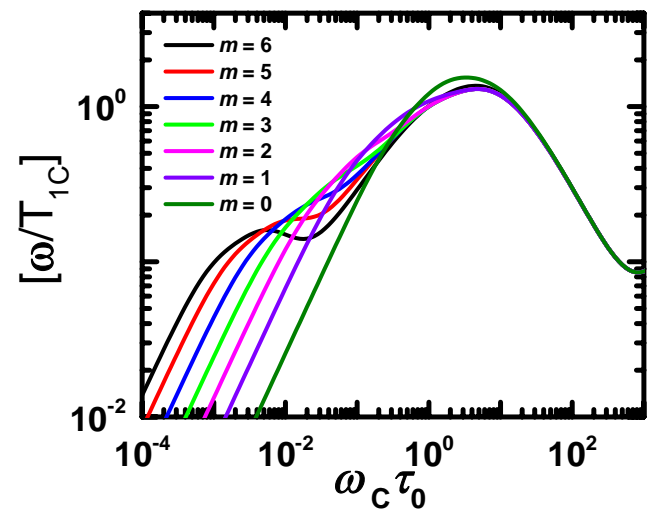

(d) FSD, $G=7$

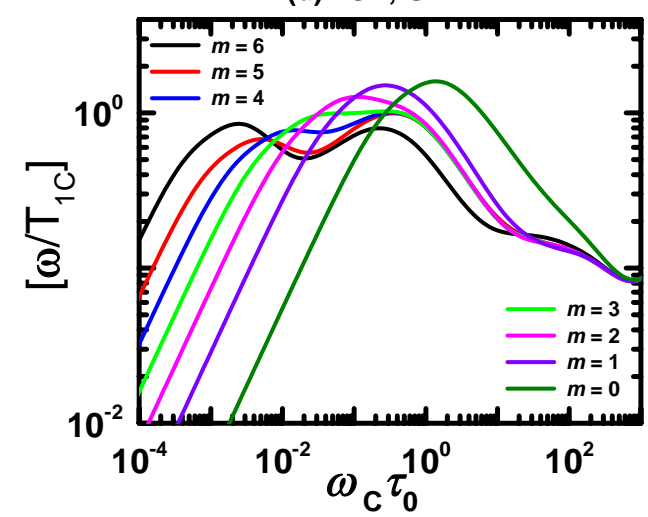

Figure 7. Reduced spin-lattice relaxation rates of ${ }^{1} \mathrm{H}(a, c)$ and ${ }^{13} \mathrm{C}(b, d)$ nuclei in the susceptibility representation for the segments (labeled by $m$ ) of functionalized flexible (FFD: $a, b)$ and semiflexible (FSD: $c, d)$ dendrimers at generation $G=7$.

C. Comparison with molecular dynamics simulations of carbosilane dendrimer melts

This subsection will compare the rate functions $\left[\omega / T_{1 \mathrm{H}}\right]$ obtained in Ref. ${ }^{28}$ using atomistic molecular dynamics (MD) simulations of carbosilane dendrimer melts (see the chemical structure of the dendrimer in Figure 2) and those of FSD obtained in this work. Carbosilane dendrimers possess a significant degree of semiflexibility. This fact was supported by simulations ${ }^{28}$ and experimental ${ }^{46}$ 
results. It should be noted that the developed theory describes the intramolecular orientational mobility, which manifests in NMR relaxation inside an individual dendrimer, in both solutions and melts. However, the rotation of the dendrimer as a whole has a significant contribution for a dendrimer in a solvent at low frequencies. In the case of dendrimer melts, the contribution of this process is minor, ${ }^{28}$ which allows us to make a direct comparison between theory and simulations. The characteristic time unit $\tau_{0}$ has been calibrated based on $\left[\omega / T_{1 \mathrm{H}}\right]$ of terminal segments $(m=0),{ }^{16}$ whose relaxation is dominated by a single process. ${ }^{13,23,28}$ As can be observed in Figure 8, the results of theory and simulations are not only qualitatively similar but also quantitatively very close. Going from a terminal segment towards the dendrimer's core $(m>0)$ one observes a separation of processes through the maximum splitting. In doing so, the position of the highfrequency maximum practically does not change, as theoretically predicted by the GD process. At the same time the low-frequency maximum shifts towards lower frequencies with growing $m$, due to the overall branch relaxation. Also, it is important to note that both in theory and simulations the amplitudes of both maxima are comparable. This fact deprives us of an explanation of the second maximum through the inner spectrum whose amplitude is much smaller in the SD model than that of the low-frequency maximum (see e.g. Ref. ${ }^{13}$ ). Even if one can speculate whether it is possible to tune the parameters (e.g., semiflexibility) in such a way that the amplitudes become comparable, such a hypothesis is rapidly rejected by looking at $\left[\omega / T_{1 \mathrm{H}}\right]$ of the terminal segments $(m=0)$. As one can see in 
Figure 8 , the position of its maximum is shifted towards high frequencies in comparison with that maximum for the inner segments, as predicted by the FSD theory and cannot be explained by the standard model: ${ }^{13}$ While for the inner segments the high-frequency maximum is due to the GD process, the relaxation of the terminal segments depends on the relaxation of a single segment whose characteristic time is smaller than that of the GD process ${ }^{36}$ but higher than that of the inner spectrum.

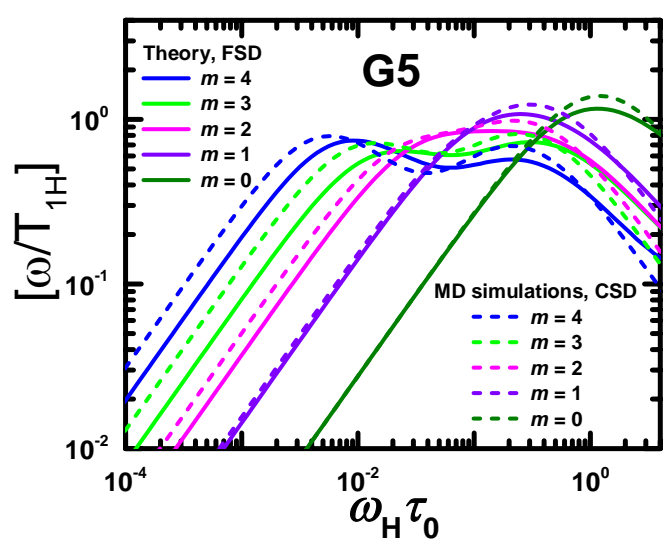

Figure 8. Frequency dependent reduced spin-lattice relaxation rate in the susceptibility representation for individual segments (labeled by $m$ ) of dendrimers obtained from the theory of functionalized semiflexible dendrimers (FSD) and from atomistic molecular dynamics simulations ${ }^{28}$ of carbosilane dendrimer (CSD) melts.

Thus, the differences between the behavior of NMR relaxation of a standard dendrimer according to the conclusions of Ref. ${ }^{13}$ and the results of the molecular dynamics simulations of carbosilane dendrimer melts ${ }^{28}$ can be explained by the fact that each $\mathrm{Si}$-atom has a $\mathrm{CH}_{3}$ group attached to it (see Figure 2), rendering the carbosilane dendrimers similar to functionalized dendrimers. Summarizing, the 
theory of FSD provides an explanation for the high-frequency behavior of NMR relaxation of carbosilane dendrimers.

\section{Conclusions}

Analytic approaches based on the viscoelastic models for polymers have recently demonstrated their effectiveness for the interpretation of the NMR relaxation of dendrimers, especially in case of melts. ${ }^{23,28}$ This work is devoted to the theory of NMR relaxation of a novel class of dendrimers containing functionalized groups that do not take part in the dendrimer's synthetic cycle..$^{30,35}$ As has been recently shown in Ref. ${ }^{36}$, the relaxation spectrum of this kind of dendrimer contains a process, the GD process, which appears due to the functionalized groups that are not present for standard dendrimers. In this work, we have shown that the GD process is a hallmark of the NMR relaxation of functionalized dendrimers. It leads to a significant maximum at high frequencies that does not depend on the topological position of the labeled segment. Exceptionally, for the terminal segments, the GD process does not play any role; as the total relaxation is dominated by the single-segment contribution. At low frequencies the functionalized dendrimers behave as the standard ones. The position of the low-frequency maximum depends on the topological remoteness of the labeled segment from the periphery. In order to see these results, the inclusion of the bending rigidity is indispensable. Otherwise, the NMR relaxation of labeled segments in a dendrimer becomes close to that of a single process relaxation, that 
is independent of both the size of the dendrimer and the location of the labeled segment.

The obtained theoretical results have been compared with those of the carbosilane dendrimer melts, recently obtained by atomistic molecular dynamics simulations. ${ }^{28}$ Not only a qualitative agreement but also even almost a quantitative one has been observed. Hereupon we could conclude that (i) the manifestation of the GD process in the NMR relaxation and its characteristic behavior is confirmed;

(ii) the theory of functionalized dendrimers has provided an interpretation of the high-frequency behavior of the NMR relaxation of carbosilane dendrimer melts.

We expect that our results will pave the way to a theory of the NMR relaxation behavior of the treelike polymers with irregularities in the branching. Also, the results show that NMR relaxation is a powerful tool for the investigation of the relationship between the structure and dynamics of dendrimers and related macromolecular compounds.

\section{Acknowledgement}

The authors thank Gregory Page (LPTMC, Sorbonne Université) for the careful reading of this manuscript. This work is supported by the Russian Science Foundation (grant No. 19-13-00087). E. L. and N. N. S. are thankful for the Researcher Mobility Grant of the Academy of Finland (No. 318608). 


\section{References}

(1) Astruc, D.; Boisselier, E.; Ornelas, C. Dendrimers Designed for Functions:

From Physical, Photophysical, and Supramolecular Properties to

Applications in Sensing, Catalysis, Molecular Electron. Chem. Rev. 2010, 110 (4), 1857-1959. https://doi.org/10.1021/cr900327d.

(2) Kesharwani, P.; Jain, K.; Jain, N. K. Dendrimer as Nanocarrier for Drug Delivery. Prog. Polym. Sci. 2014, 39 (2), 268-307. https://doi.org/http://dx.doi.org/10.1016/j.progpolymsci.2013.07.005.

(3) Gnaim, S.; Shabat, D. Quinone-Methide Species, A Gateway to Functional Molecular Systems: From Self-Immolative Dendrimers to Long-Wavelength Fluorescent Dyes. Acc. Chem. Res. 2014, 47 (10), 2970-2984. https://doi.org/10.1021/ar500179y.

(4) Scott, R. W. J.; Wilson, O. M.; Crooks, R. M. Synthesis, Characterization, and Applications of Dendrimer-Encapsulated Nanoparticles. J. Phys. Chem. B 2005, 109 (2), 692-704. https://doi.org/10.1021/jp0469665.

(5) Lo, S.-C.; Burn, P. L. Development of Dendrimers: Macromolecules for Use in Organic Light-Emitting Diodes and Solar Cells. Chem. Rev. 2007, 107 (4), 1097-1116. https://doi.org/10.1021/cr0501361.

(6) Kuang, T.; Chang, L.; Fu, D.; Yang, J.; Zhong, M.; Chen, F.; Peng, X. Improved Crystallizability and Processability of Ultra High Molecular 
Weight Polyethylene Modified by Poly(Amido Amine) Dendrimers. Polym. Eng. Sci. 2017, 57 (2), 153-160. https://doi.org/10.1002/pen.24396.

(7) Wittmer, J.; Johner, A.; Joanny, J. F.; Binder, K. Chain Desorption from a Semidilute Polymer Brush: A Monte Carlo Simulation. J. Chem. Phys. 1994, 101 (5), 4379-4390. https://doi.org/10.1063/1.468410.

(8) Kimmich, R.; Fatkullin, N. Polymer Chain Dynamics and NMR. Adv. Polym. Sci. 2004, 170, 1-113. https://doi.org/10.1007/978-3-540-40000-4_1.

(9) Rössler, E. A.; Stapf, S.; Fatkullin, N. Recent NMR Investigations on Molecular Dynamics of Polymer Melts in Bulk and in Confinement. Curr. Opin. Colloid Interface Sci. 2013, 18 (3), 173-182. https://doi.org/10.1016/j.cocis.2013.03.005.

(10) Kimmich, R. NMR - Tomography, Diffusometry, Relaxometry; SpringerVerlag, Berlin, 1997.

(11) Pinto, L. F.; Correa, J.; Martin-Pastor, M.; Riguera, R.; Fernandez-Megia, E. The Dynamics of Dendrimers by NMR Relaxation: Interpretation Pitfalls. $J$. Am. Chem. Soc. 2013, 135 (5), 1972-1977. https://doi.org/10.1021/ja311908n.

(12) Gotlib, Y. Y.; Markelov, D. A. Theory of Orientational Relaxation of Individual Specified Units in a Dendrimer. Polym. Sci. Ser. A 2007, 49 (10), 1137-1154. https://doi.org/10.1134/S0965545X07100112. 
(13) Markelov, D. A.; Dolgushev, M.; Gotlib, Y. Y.; Blumen, A. NMR Relaxation of the Orientation of Single Segments in Semiflexible Dendrimers. J. Chem. Phys. 2014, 140 (24), 244904. https://doi.org/10.1063/1.4884024.

(14) Dolgushev, M.; Schnell, S.; Markelov, D. A. Local NMR Relaxation of Dendrimers in the Presence of Hydrodynamic Interactions. Appl. Magn. Reson. 2017, 48 (7), 657-671. https://doi.org/10.1007/s00723-017-0897-6.

(15) Markelov, D. A.; Lyulin, S. V; Gotlib, Y. Y.; Lyulin, A. V; Matveev, V. V; Lahderanta, E.; Darinskii, A. A. Orientational Mobility and Relaxation Spectra of Dendrimers: Theory and Computer Simulation. J. Chem. Phys. 2009, 130 (4), 044907. https://doi.org/10.1063/1.3063116.

(16) Markelov, D. A.; Falkovich, S. G.; Neelov, I. M.; Ilyash, M. Y.; Matveev, V. V.; Lähderanta, E.; Ingman, P.; Darinskii, A. A. Molecular Dynamics Simulation of Spin-Lattice NMR Relaxation in Poly-L-Lysine Dendrimers: Manifestation of the Semiflexibility Effect. Phys. Chem. Chem. Phys. 2015, 17 (5), 3214-3226. https://doi.org/10.1039/C4CP04825C.

(17) Shavykin, O. V; Neelov, I. M.; Darinskii, A. A. Is the Manifestation of the Local Dynamics in the Spinlattice NMR Relaxation in Dendrimers Sensitive to Excluded Volume Interactions? Phys. Chem. Chem. Phys. 2016, 18, 24307-24317. https://doi.org/10.1039/C6CP01520D.

(18) Novoa-Carballal, R.; Sawen, E.; Fernandez-Megia, E.; Correa, J.; Riguera, 
R.; Widmalm, G. The Dynamics of GATG Glycodendrimers by NMR Diffusion and Quantitative 13C Relaxation. Phys. Chem. Chem. Phys. 2010, 12 (25), 6587-6589. https://doi.org/10.1039/C003645P.

(19) Pinto, L. F.; Riguera, R.; Fernandez-Megia, E. Stepwise Filtering of the Internal Layers of Dendrimers by Transverse-Relaxation-Edited NMR. $J$. Am. Chem. Soc. 2013, 135 (31), 11513-11516.

https://doi.org/10.1021/ja4059348.

(20) Sheveleva, N. N.; Markelov, D. A.; Vovk, M. A.; Mikhailova, M. E.; Tarasenko, I. I.; Neelov, I. M.; Lähderanta, E. NMR Studies of Excluded Volume Interactions in Peptide Dendrimers. Sci. Rep. 2018, 8 (1), 8916. https://doi.org/10.1038/s41598-018-27063-3.

(21) Mohamed, F.; Hofmann, M.; Pötzschner, B.; Fatkullin, N.; Rössler, E. A. Dynamics of PPI Dendrimers: A Study by Dielectric and H-2 NMR Spectroscopy and by Field-Cycling H-1 NMR Relaxometry. Macromolecules 2015, 48 (10), 3294-3302. https://doi.org/10.1021/acs.macromol.5b00486.

(22) Hofmann, M.; Gainaru, C.; Cetinkaya, B.; Valiullin, R.; Fatkullin, N.; Rössler, E. A. Field-Cycling Relaxometry as a Molecular Rheology Technique: Common Analysis of NMR, Shear Modulus and Dielectric Loss Data of Polymers vs Dendrimers. Macromolecules 2015, 48 (20), 75217534. https://doi.org/10.1021/acs.macromol.5b01805.

(23) Markelov, D. A.; Dolgushev, M.; Lähderanta, E. Chapter One - NMR 
Relaxation in Dendrimers. In Annual Reports on NMR Spectroscopy; G.A.

Webb, Ed.; Academic Press, 2017; Vol. 91, pp 1-66.

https://doi.org/http://dx.doi.org/10.1016/bs.arnmr.2016.11.001.

(24) Gotlib, Y. Y.; Markelov, D. A. Theory of the Relaxation Spectrum of a Dendrimer Macromolecule. Polym. Sci. Ser. A 2002, 44 (12), 1341-1350.

(25) Cai, C.; Chen, Z. Y. Rouse Dynamics of a Dendrimer Model in the $\vartheta$ Condition. Macromolecules 1997, 30 (17), 5104-5117. https://doi.org/10.1021/ma970059z.

(26) Gurtovenko, A. A.; Markelov, D. A.; Gotlib, Y. Y.; Blumen, A. Dynamics of Dendrimer-Based Polymer Networks. J. Chem. Phys. 2003, 119 (14), 75797590. https://doi.org/10.1063/1.1606675.

(27) Kelly, J.; Grosberg, A. Y.; Bruinsma, R. Generalized Flory Theory for Rotational Symmetry Breaking of Complex Macromolecules. Phys. Rev. Lett. 2019, 122 (12), 128003. https://doi.org/10.1103/PhysRevLett.122.128003.

(28) Markelov, D. A.; Shishkin, A. N.; Matveev, V. V; Penkova, A. V; Lähderanta, E.; Chizhik, V. I. Orientational Mobility in Dendrimer Melts: Molecular Dynamics Simulations. Macromolecules 2016, 49 (23), $9247-$ 9257. https://doi.org/10.1021/acs.macromol.6b01502.

(29) Dolgushev, M.; Markelov, D. A.; Lähderanta, E. Linear Viscoelasticity of 
Carbosilane Dendrimer Melts. Macromolecules 2019, 52 (6), 2542-2547. https://doi.org/10.1021/acs.macromol.8b02250.

(30) Sowinska, M.; Urbanczyk-Lipkowska, Z. Advances in the Chemistry of Dendrimers. New J. Chem. 2014, 38 (6), 2168-2203. https://doi.org/10.1039/C3NJ01239E.

(31) Schlüter, A. D. A Covalent Chemistry Approach to Giant Macromolecules with Cylindrical Shape and an Engineerable Interior and Surface BT Functional Molecular Nanostructures; Schlüter, A. D., Ed.; Springer Berlin Heidelberg: Berlin, Heidelberg, 2005; pp 151-191. https://doi.org/10.1007/b98168.

(32) Lederer, A.; Burchard, W.; Hartmann, T.; Haataja, J. S.; Houbenov, N.; Janke, A.; Friedel, P.; Schweins, R.; Lindner, P. Dendronized Hyperbranched Macromolecules: Soft Matter with a Novel Type of Segmental Distribution. Angew. Chemie Int. Ed. 2015, 54 (43), 12578-12583. https://doi.org/10.1002/anie.201504059.

(33) Antoni, P.; Hed, Y.; Nordberg, A.; Nyström, D.; von Holst, H.; Hult, A.; Malkoch, M. Bifunctional Dendrimers: From Robust Synthesis and Accelerated One-Pot Postfunctionalization Strategy to Potential Applications. Angew. Chemie Int. Ed. 2009, 48 (12), 2126-2130. https://doi.org/10.1002/anie.200804987.

(34) Kang, T.; Amir, R. J.; Khan, A.; Ohshimizu, K.; Hunt, J. N.; Sivanandan, K.; 
Montañez, M. I.; Malkoch, M.; Ueda, M.; Hawker, C. J. Facile Access to Internally Functionalized Dendrimers through Efficient and Orthogonal Click Reactions. Chem. Commun. 2010, 46 (9), 1556-1558. https://doi.org/10.1039/B921598K.

(35) Gadwal, I.; Khan, A. Multiply Functionalized Dendrimers: Protective-GroupFree Synthesis through Sequential Thiol-Epoxy 'Click' Chemistry and Esterification Reaction. RSC Adv. 2015, 5 (55), 43961-43964. https://doi.org/10.1039/C5RA05820A.

(36) Grimm, J.; Dolgushev, M. Dynamics of Internally Functionalized Dendrimers. Phys. Chem. Chem. Phys. 2016, 18 (28), 19050-19061. https://doi.org/10.1039/C6CP02406H.

(37) Voit, B. I.; Lederer, A. Hyperbranched and Highly Branched Polymer Architectures - Synthetic Strategies and Major Characterization Aspects. Chem. Rev. 2009, 109 (11), 5924-5973. https://doi.org/10.1021/cr900068q.

(38) Dolgushev, M.; Blumen, A. Dynamics of Semiflexible Treelike Polymeric Networks. J. Chem. Phys. 2009, 131 (4), 044905. https://doi.org/10.1063/1.3184797.

(39) Doi, M.; Edwards, S. F. The Theory of Polymer Dynamics; Oxford University Press, Oxford, 1988.

(40) Fürstenberg, F.; Dolgushev, M.; Blumen, A. Analytical Model for the 
Dynamics of Semiflexible Dendritic Polymers. J. Chem. Phys. 2012, 136 (15), 154904. https://doi.org/10.1063/1.3703757.

(41) Perico, A.; Guenza, M. Viscoelastic Relaxation of Segment Orientation in Dilute Polymer Solutions. J. Chem. Phys. 1985, 83 (6), 3103-3109. https://doi.org/10.1063/1.449216.

(42) Kruk, D.; Herrmann, A.; Rössler, E. A. Field-Cycling NMR Relaxometry of Viscous Liquids and Polymers. Prog. Nucl. Magn. Reson. Spectrosc. 2012, 63, 33-64. https://doi.org/https://doi.org/10.1016/j.pnmrs.2011.08.001.

(43) Kariyo, S.; Gainaru, C.; Schick, H.; Brodin, A.; Novikov, V. N.; Rössler, E. A. From a Simple Liquid to a Polymer Melt: NMR Relaxometry Study of Polybutadiene. Phys. Rev. Lett. 2006, 97 (20), 207803. https://doi.org/10.1103/PhysRevLett.97.207803.

(44) Chizhik, V. I.; Chernyshev, Y. S.; Donets, A. V.; Frolov, V. V.; Komolkin, A. V.; Shelyapina, M. G. Magnetic Resonance and Its Applications; Springer: Cham, 2014. https://doi.org/10.1007/978-3-319-05299-1.

(45) Matveev, V. V; Markelov, D. A.; Brui, E. A.; Chizhik, V. I.; Ingman, P.; Lahderanta, E. C-13 NMR Relaxation and Reorientation Dynamics in Imidazolium-Based Ionic Liquids: Revising Interpretation. Phys. Chem. Chem. Phys. 2014, 16 (22), 10480-10484. https://doi.org/10.1039/c4cp00637b. 
(46) Markelov, D. A.; Matveev, V. V.; Ingman, P.; Nikolaeva, M. N.; Lähderanta, E.; Shevelev, V. A.; Boiko, N. I. NMR Studies of Carbosilane Dendrimer with Terminal Mesogenic Groups. J. Phys. Chem. B 2010, 114 (12), $4159-$ 4165. https://doi.org/10.1021/jp909658v. 
for Table of Contents use only

\section{NMR Relaxation of Functionalized Dendrimers}

Nadezhda N. Sheveleva, Maxim Dolgushev, Erkki Lähderanta, Denis A. Markelov

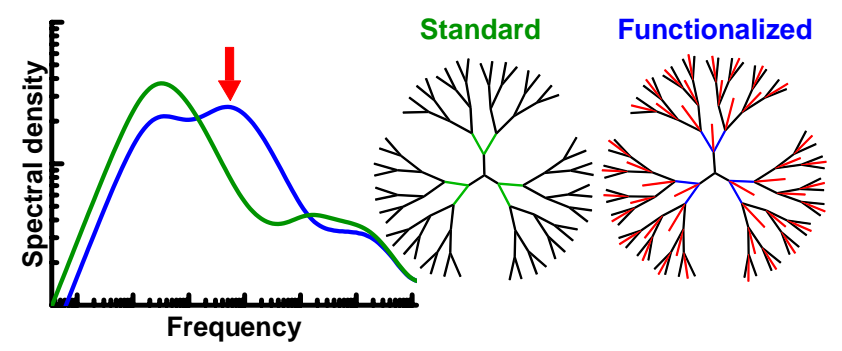

